\title{
From normal to metastases-a whole gamut of pancreatic organoids
}

$\mathrm{P}$ rogress in the early detection and treatment of pancreatic cancer has so far been hampered by the lack of relevant and tractable experimental model systems, according to Professor David Tuveson. A transatlantic collaboration between Tuveson and Professor Hans Clevers' groups published in Cell describes the development of pancreatic organoids covering normal, premalignant and malignant stages of cancer progression. Subsequent genetic and protein profiling revealed new leads on cancer biomarkers.

Organoid models are 3D in vitro culture systems that are reported to bridge the gap in preclinical research between simplistic $2 \mathrm{D}$ in vitro culture models and time-consuming and costly xenograft or genetically engineered mouse models. "Therefore, we sought to create a new [organoid] model system that would facilitate advances in our ability to diagnose and treat patients with pancreatic cancer," explains Clevers.

Initially, organoids were derived from the pancreatic ductal cells of normal mice and mice genetically programmed to develop premalignant and malignant stages of pancreatic carcinogenesis. A collection of normal $(\mathrm{mN})$ and pancreatic intraepithelial neoplasm (PanIN, mP) organoids were generated that can be continuously propagated (for $>20$ passages) and that survive cryopreservation. These characteristics make them a fairly easy and, therefore, useful tool to work with in the laboratory.

Histological analysis of the tissue from orthotopically transplanted $\mathrm{mN}$ organoids revealed the development of ductal structures, which persisted for up to 1 month. Tissues originating from transplanted $\mathrm{mP}$ organoids were found to have many features of mouse PanINs, including: expression of $M u c 5 a c$, a mucin gene associated with mouse PanIN lesions; increased proliferation; and a large stromal component.
Next, the researchers developed organoids from mouse primary (mT) and metastatic $(\mathrm{mM})$ pancreatic tumour tissue (see image). Lesions generated by orthotopic transplantation of $\mathrm{mT}$ organoids also resembled mouse PanINs, but, over 1-6 months, developed into primary and invasive pancreatic ductal adenocarcinomas. Importantly, the engraftment efficiency of mT organoids was $85 \%$ in $\mathrm{C} 57 \mathrm{Bl} / 6$ mice and $91.7 \%$ in immunodeficient nude mice. $\mathrm{mM}$ organoids formed invasive pancreatic ductal adenocarcinomas within 1 month of implantation.

In contrast to xenografts generated from 2D immortalized pancreatic cell lines, the mouse pancreatic organoid model provides a way of studying all stages of disease development and recapitulates aspects of human cancer such as the prominent stromal reaction and low vascular density. "Previously, it had not been possible to culture pre-malignant stages of disease," remarks Tuveson. "This advance will enable evaluation of these earlier stages of pancreatic cancer for putative biomarkers." Indeed, analysis of the mouse organoids highlighted an upregulation of the nucleoporin family at the RNA and protein level in $\mathrm{mT}$ relative to $\mathrm{mN}$ organoids. Nuclear transport might therefore represent a pathway that is similarly disrupted in human disease progression.

A human model was also generated using surgically resected samples of normal and tumour pancreatic tissue. However, $85 \%$ of patients are not eligible for surgical resection and so the authors decided to try and generate organoids from endoscopic fine needle aspiration biopsy samples, which can be obtained from more patients than those from surgery. By not enzymatically digesting the samples before 3D culturing, they were able to generate viable organoids. Genomic profiling of the normal and

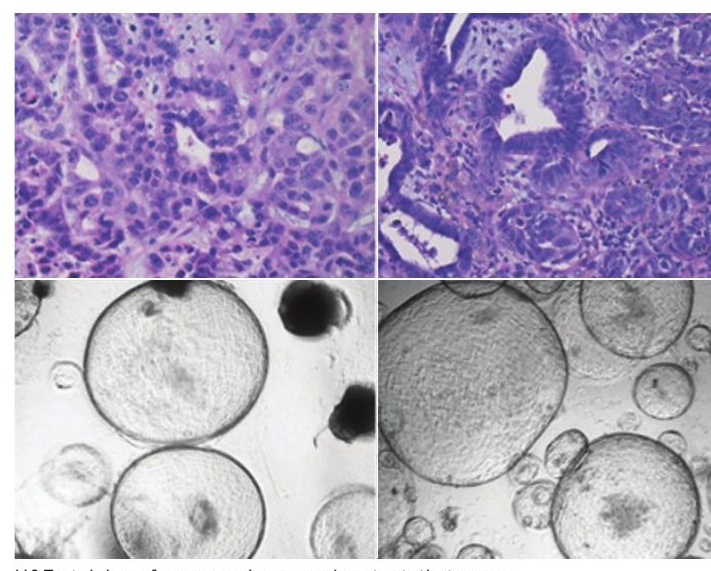

H\&E staining of mouse primary and metastatic tumour tissue (top) and the organoids derived from them (bottom). Image courtesy of Chang-il Hwang.

tumour organoids identified many changes, including mutations in KRAS, TP53, SMAD4 and DCC. The authors believe that using fine needle aspiration biopsy samples might enable personalized approaches to pancreatic cancer treatment as organoid cultures can be generated efficiently in weeks. By contrast, 2D cultures derived from biopsy samples can be difficult to establish and xenografts from biopsy samples can require up to a year to grow enough material for further experimentation.

Transplantation of the human organoids into immunodeficient nude mice had a low success rate for the normal organoids $(n=2 / 23)$ but a high success rate for the tumour organoids $(n=9 / 12)$. Similar to the mouse organoids, the human tumour organoids advanced to poorly differentiated, invasive carcinomas.

Going forward, the groups hope to create an organoid repository for worldwide distribution to help advance pancreatic cancer research.

Gillian Patman

Original article Boj, S. F. et al. Organoid models of human and mouse ductal pancreatic cancer. Cell doi:10.1016/ j.cell.2014.12.021 